\title{
On maximum signless Laplacian Estrada index of graphs with given parameters
}

\author{
Hamid Reza Ellahi \\ Department of Mathematics, Faculty of Sciences, University of Qom, 37161-46611, \\ Qom, I. R. Iran \\ Gholam Hossein Fath-Tabar * \\ Department of Pure Mathematics, Faculty of Mathematical Sciences, University of \\ Kashan, 87317-51167, Kashan, I. R. Iran \\ Ahmad Gholami, Ramin Nasiri \\ Department of Mathematics, Faculty of Sciences, University of Qom, 37161-46611, \\ Qom, I. R. Iran
}

Received 21 April 2015, accepted 15 December 2015, published online 19 July 2016

\begin{abstract}
For a simple graph $G$ on $n$ vertices, the signless Laplacian Estrada index is defined as $\operatorname{SLEE}(G)=\sum_{i=1}^{n} e^{q_{i}}$, where $q_{1}, q_{2}, \ldots, q_{n}$ are the eigenvalues of the signless Laplacian matrix of $G$. In this paper, the unique graph on $n$ vertices with maximum signless Laplacian Estrada index is determined among graphs with given number of cut edges, pendent vertices, (vertex) connectivity and edge connectivity, respectively.

Keywords: Signless Laplacian Estrada index, semi-edge walk, cut edge, vertex connectivity, edge connectivity.
\end{abstract}

Math. Subj. Class.: 05C50, 05C12, 05C35.

\section{Introduction}

Throughout this paper, each graph, say $G$, is simple with its vertex and edge sets $V(G)$ and $E(G)$, respectively. If $|V(G)|=n$, then $G$ is considered as an $n$-vertex graph. Let $u$ and $v$ be two vertices of $G$. We say that $u$ is a neighbor of $v$, if they are joined together in $G$. The

*Financially supported by University of Kashan (Grant No. 504631/6).

E-mail addresses: H.R.Ellahi@Gmail.com (Hamid Reza Ellahi), Fathtabar@kashanu.ac.ir (Gholam Hossein Fath-Tabar), Gholami@Kashanu.ac.ir (Ahmad Gholami), R.Nasiri.82@Gmail.com (Ramin Nasiri) 
number of neighbors of $v$ in $G$ is called the degree of $v$, and denoted by $d_{G}(v)$. The vertex $v$ of $G$ is referred to as a pendent vertex if it has only one neighbor (i.e. $d_{G}(v)=1$ ). A cut edge of a (connected) graph $G$ is an edge whose deletion disconnects $G$. The vertex (resp. edge) connectivity of $G$ is the minimum number of vertices (resp. edges) which need to be removed to disconnect $G$ or convert it to a single vertex.

Let $\mathbf{A}(G)$ be the adjacency matrix of $G$ and $\mathbf{D}(G)=\left[d_{i j}\right]_{n \times n}$ be the diagonal matrix, where the element $d_{i j}$ is equal to $d_{G}\left(v_{i}\right)$ if $i=j$, and 0 otherwise. The Laplacian matrix and signless Laplacian matrix of $G$ are denoted by $\mathbf{L}(G)$ and $\mathbf{Q}(G)$, respectively, where $\mathbf{L}(G)=\mathbf{D}(G)-\mathbf{A}(G)$ and $\mathbf{Q}(G)=\mathbf{D}(G)+\mathbf{A}(G)$ (see [7,22]). We represent the eigenvalues of $\mathbf{A}(G), \mathbf{L}(G)$ and $\mathbf{Q}(G)$ by $\lambda_{1}, \lambda_{2}, \ldots, \lambda_{n} ; \mu_{1}, \mu_{2}, \ldots, \mu_{n}$; and $q_{1}, q_{2}, \ldots, q_{n}$, respectively.

Estrada [11,12], for the first time, defined a graph-spectrum-based invariant and named it Estrada index, which is as follows:

$$
E E(G)=\sum_{i=1}^{n} e^{\lambda_{i}}
$$

Fath-Tabar et al. [13] proposed the Laplacian Estrada index (after here LEE), in full analogy with Estrada index as

$$
L E E(G)=\sum_{i=1}^{n} e^{\mu_{i}}
$$

Estrada and Laplacian Estrada indices have been studied in a large variety of problems. In the mathematical literature, there are two types of problems in almost all papers researching on such indices: finding bounds for the index (e.g. [1, 4, 16, 20, 24]), and determining extremal graphs with respect to the index (e.g. [10,21,23, 25]).

Ayyaswamy et al. [2] defined the signless Laplacian Estrada index (SLEE) as

$$
\operatorname{SLEE}(G)=\sum_{i=1}^{n} e^{q_{i}} .
$$

They also established lower and upper bounds for $S L E E$ in terms of the number of vertices and edges. They showed that for any graph $G$ on $n$ vertices and $m$ edges,

$$
\sqrt{n+4 m+n(n-1) e^{\frac{4 m}{n}}} \leq \operatorname{SLEE}(G) \leq n-1+e^{\sqrt{\left(n^{2}-n+2 m\right) m}}
$$

with equality on both sides if and only if $G$ is empty. In the same sense, Binthiya et al. [5] established upper bound for $S L E E$ in terms of the vertex connectivity of graph and the specific corresponding extremal graph. They find that [5, Theorem 3.1.] for each $(n, m)$ graph $G$ with vertex connectivity $\kappa$,

$$
S L E E(G) \leq \kappa e^{n-2}+(n-\kappa-2) e^{n-3}+e^{2 n+\kappa-4} .
$$

It is well-known that the Laplacian and signless Laplacian spectra of bipartite graphs coincide (see [14,15]). Thus, for a bipartite graph $G, S L E E(G)=L E E(G)$. Chemically, since the vast majority of molecular graphs are bipartite, we can use the provided statements in $S L E E$ for $L E E$, and vice versa. However, the interesting case occurs when $S L E E$ 
and $L E E$ differ, namely, in fullerenes, fluoranthenes and other non-alternant conjugated species [3, 9, 17-19].

Moreover, Cvetković et al. [8] gathered many reasons about advantage of studying Qspectra. They found that the signless Laplacian spectra perform better in comparison to spectra of other commonly used graph matrices, say the Laplacian, or the Seidel matrix. Also, they expressed that among generalized adjacency matrices, i.e. matrices which are a linear combination of $\mathbf{A}(G), \mathbf{J}$ (the all-ones matrix) and $\mathbf{I}$ (the identity matrix) with a non-zero coefficient for $\mathbf{A}(G)$, the signless Laplacian seems to be the most convenient for use in studying graph properties.

The goal of this paper is to find the unique extremal graph with maximum SLEE among all $n$-vertex graphs with given number of cut edges, pendent vertices, (vertex) connectivity, or edge connectivity.

Our main results are the following two theorems:

Theorem 1.1. Let $0 \leq p<n$, and $G_{n, p}$ be the graph obtained by attaching pendent vertices to one vertex of complete graph $K_{n-p}$ (see Figure 1). Then, up to isomorphism, we have:

1. Among the set of all n-vertex graphs having $p$ cut edges, $G_{n, p}$ is the unique graph with maximum SLEE.

2. Among the set of all n-vertex graphs having $p$ pendent vertices, $G_{n, p}$ is the unique graph with maximum SLEE.

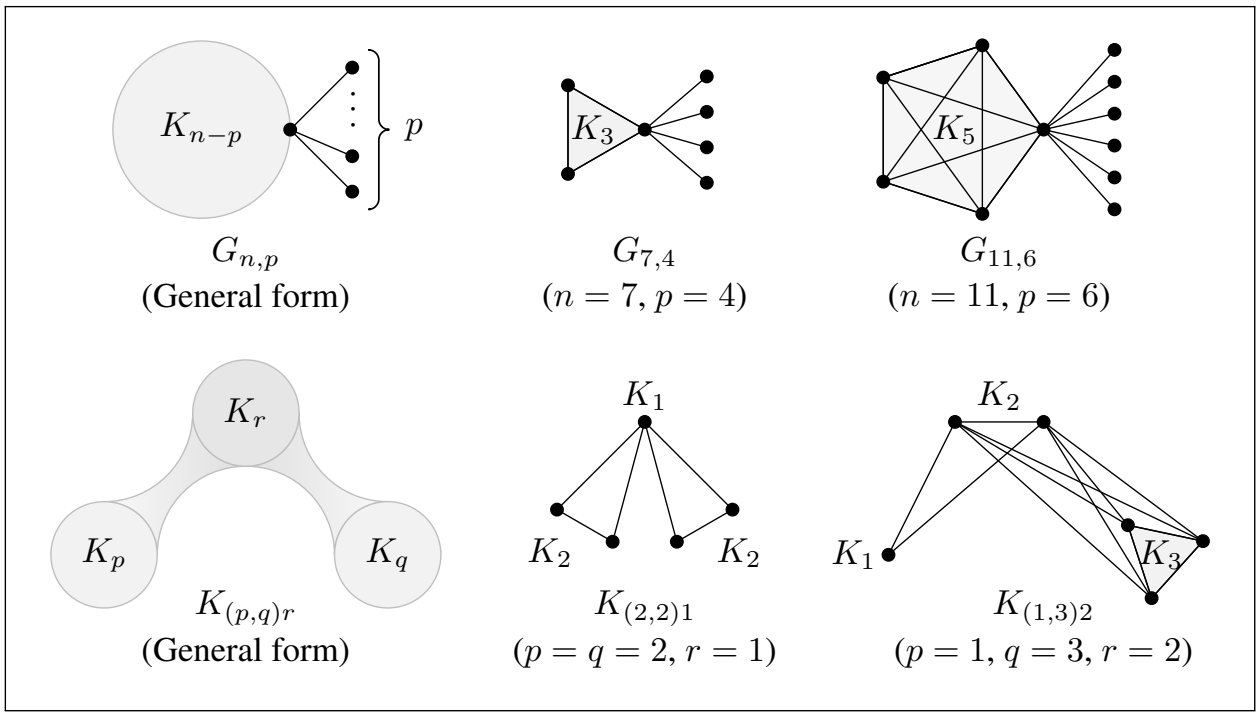

Figure 1: An illustration of the general forms of $G_{n, p}$ and $K_{(p, q) r}$, with some examples.

Theorem 1.2. Let $p, q$ and $r$ be three non-negative integers, and let $K_{(p, q) r}$ be the graph obtained from three vertex-disjoint complete graphs $K_{p}, K_{q}$ and $K_{r}$, by attaching any vertex of $K_{r}$ to all vertices of both $K_{p}$ and $K_{q}$ (see Figure 1). Then, up to isomorphism, we have: 
1. Among the set of all $n$-vertex graphs with vertex connectivity $\kappa$, where $0 \leq \kappa \leq n-1$, $K_{(n-1-\kappa, 1) \kappa}$ is the unique graph with maximum SLEE.

2. Among the set of all $n$-vertex graphs with edge connectivity $\kappa^{\prime}$, where $0 \leq \kappa^{\prime} \leq n-1$, $K_{\left(n-1-\kappa^{\prime}, 1\right) \kappa^{\prime}}$ is the unique graph with maximum $S L E E$.

\section{Preliminaries and lemmas}

Before proving our main theorems, we shall provide some fundamental definitions and propositions. In this section, we first declare some basic and useful notations, definitions and a proved theorem; afterward, we propose three effective lemmas to prove Theorems 1.1 and 1.2.

Denote by $T_{k}(G)$ the $k$-th signless Laplacian spectral moment of the graph $G$, i.e., $T_{k}(G)=\sum_{i=1}^{n} q_{i}^{k}$. By applying the Taylor expansion to the function $e^{x}$, we have:

$$
\operatorname{SLEE}(G)=\sum_{k \geq 0} \frac{T_{k}(G)}{k !}
$$

Moreover, by the following definition and theorem, we can easily compare the $S L E E$ 's of some graphs.

Definition 2.1. [8] A semi-edge walk of length $k$ in a graph $G$ is an alternating sequence $W=v_{1} e_{1} v_{2} e_{2} \cdots v_{k} e_{k} v_{k+1}$ of vertices $v_{1}, v_{2}, \ldots, v_{k}, v_{k+1}$ and edges $e_{1}, e_{2}, \ldots, e_{k}$ such that the vertices $v_{i}$ and $v_{i+1}$ are end-vertices (not necessarily distinct) of the edge $e_{i}$, for any $i=1,2, \ldots, k$. If $v_{1}=v_{k+1}$, then $W$ is said to be a closed semi-edge walk.

Theorem 2.2. [8] The signless Laplacian spectral moment $T_{k}$ is equal to the number of closed semi-edge walks of length $k$.

Let $G$ and $G^{\prime}$ be two graphs, and $x, y \in V(G)$, and $x^{\prime}, y^{\prime} \in V\left(G^{\prime}\right)$. Denoting by $S W_{k}(G ; x, y)$, the set of all semi-edge walks of length $k$ in $G$, each of which starts at vertex $x$ and ends at vertex $y$. Note that $\left|S W_{k}(G ; x, y)\right|=\left|S W_{k}(G ; y, x)\right|$ for any $x, y \in V(G)$. For convenience, we may denote $S W_{k}(G ; x, x)$ by $S W_{k}(G ; x)$. The notation $S W_{k}(G)$ will be used as the set of all closed semi-edge walks of length $k$ in $G$, i.e. $S W_{k}(G)=\bigcup_{x \in V(G)} S W_{k}(G ; x)$. We will use the notation $(G ; x, y) \preceq_{s}\left(G^{\prime} ; x^{\prime}, y^{\prime}\right)$ when for any $k \geq 0,\left|S W_{k}(G ; x, y)\right| \leq\left|S W_{k}\left(G^{\prime} ; x^{\prime}, y^{\prime}\right)\right|$. Moreover, if $(G ; x, y) \preceq_{s}\left(G^{\prime} ; x^{\prime}, y^{\prime}\right)$, and there exists some $k_{0}$ where $\left|S W_{k_{0}}(G ; x, y)\right|<\left|S W_{k_{0}}\left(G^{\prime} ; x^{\prime}, y^{\prime}\right)\right|$, then we will write $(G ; x, y) \prec_{s}\left(G^{\prime} ; x^{\prime}, y^{\prime}\right)$. We abbreviate $(G ; x, x) \preceq_{s}\left(G^{\prime} ; x^{\prime}, x^{\prime}\right)$ as $(G ; x) \preceq_{s}\left(G^{\prime} ; x^{\prime}\right)$, and $(G ; x, x) \prec_{s}\left(G^{\prime} ; x^{\prime}, x^{\prime}\right)$ as $(G ; x) \prec_{s}\left(G^{\prime} ; x^{\prime}\right)$.

Indeed, by using the above notations, we can restate the Theorem 2.2 as:

$$
T_{k}(G)=\left|S W_{k}(G ; x)\right|=\left|\bigcup_{x \in V(G)} S W_{k}(G ; x)\right|=\sum_{x \in V(G)}\left|S W_{k}(G ; x)\right| .
$$

Let $G$ be a graph and $E$ be a set of edges. If $E \subseteq E(G)$, then we write $G-E$ for the graph obtained from $G$ by removing all of its edges in $E$. Also, if $E \subseteq E(\bar{G})$, then we denote by $G+E$ the graph obtained from $G$ by adding all of edges in $E$ to the graph. For convenience, we set $G+e$ for $G+\{e\}$. The next result immediately follows from equations (2.1) and (2.2). 
Lemma 2.3. Let $G$ be a graph. If e is an edge such that e $\notin E(G)$, then $\operatorname{SLEE}(G)<$ $\operatorname{SLEE}(G+e)$.

Under the terms of the following lemma, we may call the vertices $w_{1}, \ldots, w_{r}$ as transferred neighbors, and the graph $G$ as transfer route graph. Also, we may say that $G_{u}$ is obtained from $G_{v}$ by transferring some neighbors of $v$ to the set of neighbors of $u$.

Lemma 2.4. Let $G$ be a graph and $v, u, w_{1}, w_{2}, \ldots, w_{r} \in V(G)$. Suppose that $E_{v}=$ $\left\{e_{1}=v w_{1}, \ldots, e_{r}=v w_{r}\right\}$ and $E_{u}=\left\{\bar{e}_{1}=u w_{1}, \ldots, \bar{e}_{r}=u w_{r}\right\}$ are subsets of edges, that are not in $G$ (i.e. $e_{i}, \bar{e}_{i} \notin E(G)$, for $\left.i=1,2, \ldots, r\right)$. Let $G_{u} \cong G+E_{u}$ and $G_{v} \cong G+E_{v}$. If $(G ; v) \prec_{s}(G ; u)$, and $\left(G ; w_{i}, v\right) \preceq_{s}\left(G ; w_{i}, u\right)$ for each $i=1,2, \ldots, r$, then $\operatorname{SLEE}\left(G_{v}\right)<\operatorname{SLEE}\left(G_{u}\right)$, where $G_{u}$ and $G_{v}$ are shown in Figure 2.

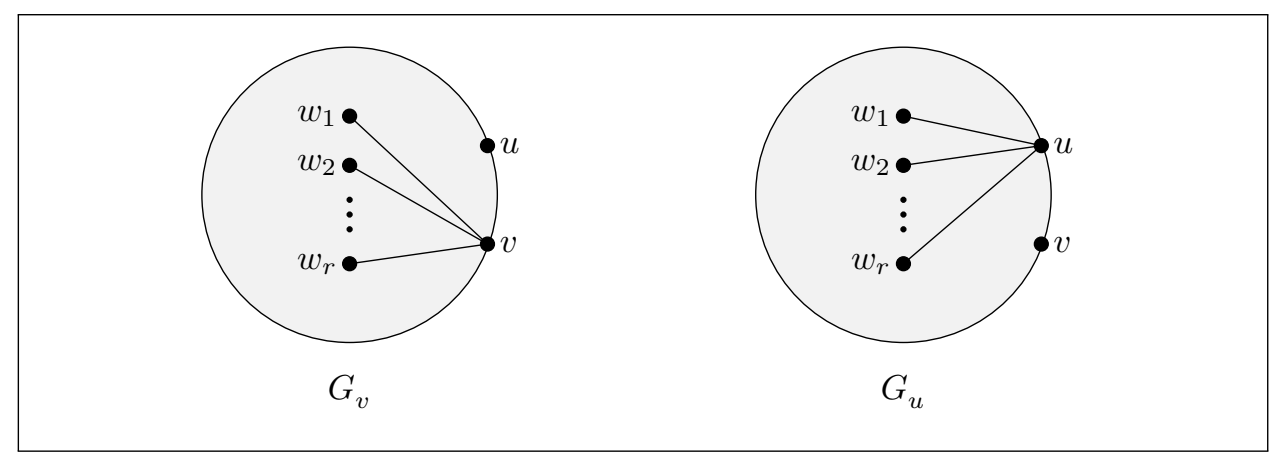

Figure 2: An illustration of the graphs $G_{v}$ and $G_{u}$ in Lemma 2.4.

Proof. Since $(G ; v) \prec_{s}(G ; u)$, there exists an injection

$$
f_{k}: S W_{k}(G ; v) \rightarrow S W_{k}(G ; u)
$$

for each $k \geq 0$. Similarly, $\left(G ; w_{i}, v\right) \preceq_{s}\left(G ; w_{i}, u\right)$ implies that there exist following injections for each $i=1,2, \ldots, r$, and $k \geq 0$ :

$$
\begin{aligned}
& f_{k}^{i}: S W_{k}\left(G ; w_{i}, v\right) \rightarrow S W_{k}\left(G ; w_{i}, u\right) \\
& g_{k}^{i}: S W_{k}\left(G ; v, w_{i}\right) \rightarrow S W_{k}\left(G ; u, w_{i}\right)
\end{aligned}
$$

For any $W \in S W_{k}(G ; x, y)$ where $x, y \in\left\{v, w_{1}, \ldots, w_{r}\right\}$, let $\bar{W}$ be as follows:

1) If $k=0$ and $W=w_{t}$, where $t \in\{1, \ldots, r\}$, then $\bar{W}=W$.

2) If $W \in S W_{k}(G ; v)$, then $\bar{W}=f_{k}(W)$.

3) If $W \in S W_{k}\left(G ; w_{t}, v\right)$, then $\bar{W}=f_{k}^{t}(W)$.

4) If $W \in S W_{k}\left(G ; v, w_{t}\right)$, then $\bar{W}=g_{k}^{t}(W)$.

5) If $W \in S W_{k}\left(G ; w_{t}, w_{j}\right)$, where $t, j \in\{1, \ldots, r\}$, then $\bar{W}=W$. 
To prove the statement, it is enough to show that $T_{k}\left(G_{v}\right) \leq T_{k}\left(G_{u}\right)$, and there exists $k_{0}$ such that inequality is strict.

If $W \in S W_{k}\left(G_{v}\right)$, then $W$ can be decomposed uniquely to $s+1$ sections as $W=$ $W_{1} e_{j_{1}} W_{2} e_{j_{2}} W_{3} \cdots W_{s} e_{j_{s}} W_{s+1}$, where $e_{j_{1}}, \ldots, e_{j_{s}} \in E_{v}$, and for each $i \in\{2,3, \ldots, s\}$, there are $x_{i}, y_{i} \in\left\{v, w_{1}, \ldots, w_{r}\right\}$ such that $W_{i} \in S W_{k_{i}}\left(G ; x_{i}, y_{i}\right)$. Moreover, since $W$ is closed, for some $x^{\prime}, y^{\prime} \in\left\{v, w_{1}, \ldots, w_{r}\right\}, W^{\prime}=W_{s+1} W_{1} \in S W_{k^{\prime}}\left(G ; x^{\prime}, y^{\prime}\right)$ where $k^{\prime}=k_{1}+k_{s+1}$. Therefore, we can uniquely decompose $\bar{W}^{\prime}$ to $\bar{W}_{s+1} \bar{W}_{1}$, where $\bar{W}_{s+1}$ is a semi-edge walk of length $k_{s+1}$ in $G$, starting at $x$ and ending at $z$, and $\bar{W}_{1}$ is a semi-edge walk of length $k_{1}$ in $G$, starting at $z$ and ending at $y$, for some $z \in V(G)$ and $x, y \in\left\{u, w_{1}, \ldots, w_{r}\right\}$.

Now, one can show that the map $h_{k}: S W_{k}\left(G_{v}\right) \rightarrow S W_{k}\left(G_{u}\right)$ defined by the rule

$$
\begin{aligned}
h_{k}(W) & =h_{k}\left(W_{1} e_{j_{1}} W_{2} e_{j_{2}} W_{3} \cdots W_{s} e_{j_{s}} W_{s+1}\right) \\
& =\bar{W}_{1} \bar{e}_{j_{1}} \bar{W}_{2} \bar{e}_{j_{2}} \bar{W}_{3} \cdots \bar{W}_{s} \bar{e}_{j_{s}} \bar{W}_{s+1}
\end{aligned}
$$

is injective, for each $k \geq 0$. Thus, $T_{k}\left(G_{v}\right) \leq T_{k}\left(G_{u}\right)$, for any $k \geq 0$.

Moreover, for some $k_{0}, f_{k_{0}}$ is not surjective, and $\left|S W_{k_{0}}(G, v)\right|<\left|S W_{k_{0}}(G, u)\right|$, i.e. $T_{k_{0}}\left(G_{v}\right)<T_{k_{0}}\left(G_{u}\right)$. Hence $\operatorname{SLEE}\left(G_{v}\right)<\operatorname{SLEE}\left(G_{u}\right)$.

The following lemma enables us to provide the necessary conditions in Lemma 2.4, and to use it to compare $S L E E$ 's of some particular graphs.

Lemma 2.5. Let $G$ be a graph and $u, v \in V(G)$. If $v$ is a pendent vertex attached to $u$, then $(G ; v) \preceq_{s}(G ; u)$, with equality if and only if $d_{G}(u)=d_{G}(v)=1$. Moreover, $(G ; w, v) \preceq_{s}(G ; w, u)$ for each $w \in V(G) \backslash\{v\}$.

Proof. The case $k=0$ is trivial. Let $k>1$ and $W=v e W^{\prime} e v \in S W_{k}(G ; v)$, where $W^{\prime}$ is a semi-edge walk of length $k-2$ in $G$. We may construct an injection $f_{k}: S W_{k}(G ; v) \rightarrow$ $S W_{k}(G ; u)$, by the rule $f_{k}(W)=u e W^{\prime} e u$. Thus $\left|S W_{k}(G ; v)\right| \leq\left|S W_{k}(G ; u)\right|$, for any $k \geq 2$. Moreover, if $d_{G}(u)>1$, then we have $\left|S W_{1}(G ; v)\right|=\bar{d}_{G}(v)=1<d_{G}(u)=$ $\left|S W_{1}(G ; u)\right|$. Recall that if $d_{G}(u)=1$, then $G$ has an automorphism, interchanges $u$ and $v$ and fixes the other vertices.

In a similar way, by changing the end of each member of $S W_{k}(G ; w, v)$ from $v$ to $u$, we find that $(G ; w, v) \preceq_{s}(G ; w, u)$ for each $w \in V(G) \backslash\{v\}$. Note that for $k=0$, $\left|S W_{0}(G ; v, v)\right|=1>0=\left|S W_{0}(G ; v, u)\right|$.

\section{The proof of Theorem 1.1}

At the end of this section, we prove our first main theorem which determines the unique extremal graph with maximum $S L E E$ among the set of all $n$-vertex graphs with given number of cut edges or pendent vertices. However, we should bring forth another lemma to this purpose.

Let us denote by $\mathcal{G}(n, p)$, the set of all graphs obtained by attaching $p$ pendent vertices to some vertices of a complete graph $K_{n-p}$, for $0 \leq p<n$.

Lemma 3.1. Suppose that $1 \leq p \leq n-3$, and $G \in \mathcal{G}(n, p)$. If $u$ and $v$ are two distinct non-pendent vertices of $G$, such that $d_{G}(v)=n-p-1<d_{G}(u)$, then $(G ; v) \prec_{s}(G ; u)$. 
Proof. Obviously, $u$ has at least one pendent neighbor and each neighbor of $v$ is $u$ itself or one of its neighbors. Thus, for each $k>1$, the map $f_{k}: S W_{k}(G ; v) \rightarrow S W_{k}(G ; u)$ defined by the rule $f_{k}\left(v e_{1} W e_{k} v\right)=u e_{1}^{\prime} W e_{k}^{\prime} u$ is well-defined and injective, where $e_{i}=v w_{i}$ and $e_{i}^{\prime}=u w_{i}$, for some neighbors $w_{i}$ of $v$ and $i \in\{1, k\}$. Therefore, $\left|S W_{k}(G ; v)\right| \leq$ $\left|S W_{k}(G ; u)\right|$, for any $k>1$. Moreover, $\left|S W_{1}(G ; v)\right|=d_{G}(v)=n-p-1<d_{G}(u)=$ $\left|S W_{1}(G ; u)\right|$ implies $(G ; v) \prec_{s}(G ; u)$.

Proof of Theorem 1.1. To prove the first part, let $G$ be an extremal $n$-vertex graph with maximum $S L E E$, having $p$ cut edges. We shall show that $G \cong G_{n, p}$.

If $p=0$, then by Lemma $2.3, G \cong K_{n} \cong G_{n, 0}$. Moreover, if $n=1$ or 2 , then $p=n-1$, and $G \cong K_{n} \cong G_{n, n-1}$. Therefore, suppose that $n \geq 3$ and $p \geq 1$.

If $E$ is the set of all cut edges in $G$, then by Lemma 2.3, all of $p+1$ connected components of $G-E$ are complete. Suppose that there exists one edge $e$ of $E$, attaching vertices $u$ and $v$ in $G$, where $d_{G}(u), d_{G}(v) \geq 2$. Let $G^{\prime}$ be the graph obtained from $G$ by transferring all neighbors of $v$ except $u$ to the set of neighbors of $u$, and $H$ be the transfer route graph. By Lemma 2.5, $(H ; v) \prec_{s}(H ; u)$. Thus, Lemma 2.4 results in $S L E E(G)<S L E E\left(G^{\prime}\right)$. This is a contradiction, because both graphs $G$ and $G^{\prime}$ have the same number of cut edges. Therefore, each cut edge incidents to a pendent vertex.

Let $V_{p}$ be a set of vertices of $G$, each of which is a pendent vertex of a cut edge such that there is no cut edge with both ends in $V_{p}$. By Lemma 2.3, $G-V_{p}$ is a complete graph on $n-p$ vertices. Thus $G$ is a graph obtained from $K_{n-p}$, by attaching $p$ pendent vertices to some vertices of $K_{n-p}$, which means $G \in \mathcal{G}(n, p)$.

If $G \neq G_{n, p}$, then there are at least two non-pendent vertices, say $u$ and $v$, such that each of them has at least one pendent neighbor. Now, by Lemmas 2.4 and 3.1 and transferring pendent neighbors of $v$ to the set of neighbors of $u$, we may get a graph with higher $S L E E$ than $G$ in $\mathcal{G}(n, p)$, which is a contradiction again. Therefore, $G \cong G_{n, p}$.

Now, to prove the second part, suppose that $G$ is an extremal $n$-vertex graph with maximum $S L E E$, having $p$ pendent vertices. If $H$ is the graph obtained from $G$, by removing all of its $p$ pendent vertices, then by Lemma 2.3, $H$ is a complete graph on $n-p$ vertices. Thus $G \in \mathcal{G}(n, p)$. Finally, the proof of this part is accomplished by using the same argument mentioned in the above paragraph.

\section{The proof of Theorem 1.2}

In this section, we are going to prove Theorem 1.2, which determines the unique extremal graph with maximum $S L E E$ among the set of all $n$-vertex graphs with given (vertex) connectivity or edge connectivity.

We start with the following lemma:

Lemma 4.1. If $2 \leq q \leq p$, and $r \geq 0$, then

$$
\operatorname{SLEE}\left(K_{(p, q) r}\right)<S L E E\left(K_{(p+q-1,1) r}\right) .
$$

Proof. Suppose that $V\left(K_{p}\right)=\left\{x_{1}, x_{2}, \ldots, x_{p}\right\}$, and $V\left(K_{q}\right)=\left\{y_{1}, y_{2}, \ldots, y_{q}\right\}$, and $V\left(K_{r}\right)=\left\{z_{1}, z_{2}, \ldots, z_{r}\right\}$. Let $G$ be the graph obtained from $K_{(p, q) r}$ by transferring neighbors $y_{2}, \ldots, y_{q}$ of $y_{1}$ to the set of neighbors of $x_{1}$, and $H$ be the transfer route graph.

If $r=0$, then obviously $\left(H ; y_{1}\right) \prec_{s}\left(H ; x_{1}\right)$. If $r>0$, then, since any neighbor of $y_{1}$ in $H$ is a neighbor of $x_{1}$, by a similar method used in the proof of Lemma 3.1, we can also show that $\left(H ; y_{1}\right) \prec_{s}\left(H ; x_{1}\right)$ and $\left(H ; y_{i}, y_{1}\right) \preceq_{s}\left(H ; y_{i}, x_{1}\right)$, for each $i \in\{2, \ldots, q\}$. 
Therefore, Lemma 2.4 implies $S L E E\left(K_{(p, q) r}\right)<S L E E(G)$. Note that, since $p \geq 2, G$ is a proper subgraph of $K_{(p+q-1,1) r}$. Thus, by Lemma 2.3,

$$
\operatorname{SLEE}\left(K_{(p, q) r}\right)<S L E E(G)<S L E E\left(K_{(p+q-1,1) r}\right) .
$$

Proof of Theorem 1.2. Note that the case $\kappa=n-1$ is trivial, because $K_{(0,1) \kappa} \cong K_{n}$ is (up to isomorphism) the unique graph with vertex connectivity $n-1$.

Let $G$ be an extremal graph with maximum $S L E E$, among $n$-vertex graphs with connectivity $\kappa$. Suppose that $S$ is a subset of $V(G)$, where $G-S$ is disconnected, and $|S|=\kappa$. By Lemma 2.3, there exist integers $p$ and $q$, such that $1 \leq q \leq p, p+q=n-\kappa$, and $G-S$ is the union of two complete components $K_{q}$ and $K_{p}$. Again, by Lemma 2.3, each vertex in $S$ is attached to another one and also to vertices of both $K_{q}$ and $K_{p}$. It means that $G \cong K_{(p, q) \kappa}$. If $q \geq 2$, then Lemma 4.1 implies $S L E E(G)<S L E E\left(K_{(p+q-1,1) \kappa}\right)$, which is a contradiction. Hence, $q=1$, and therefore $G \cong K_{(n-1-\kappa, 1) \kappa}$. This proves the first part of the theorem.

To prove the second part, we note that if $G$ is a graph with vertex and edge connectivity $\kappa$ and $\kappa^{\prime}$, respectively, then $\kappa \leq \kappa^{\prime}$ (see [6]). If $\kappa=\kappa^{\prime}$, then by previous part of the proof we have $\operatorname{SLEE}(G) \leq S L E E\left(K_{\left(n-1-\kappa^{\prime}, 1\right) \kappa^{\prime}}\right)$, and equality holds if and only if $G \cong K_{\left(n-1-\kappa^{\prime}, 1\right) \kappa^{\prime}}$. Moreover, if $\kappa<\kappa^{\prime}$ then $K_{(n-1-\kappa, 1) \kappa}$ is a proper subgraph of $K_{\left(n-1-\kappa^{\prime}, 1\right) \kappa^{\prime}}$. In this case, Lemma 2.3 and the above arguments show that

$$
S L E E(G) \leq S L E E\left(K_{(n-1-\kappa, 1) \kappa}\right)<S L E E\left(K_{\left(n-1-\kappa^{\prime}, 1\right) \kappa^{\prime}}\right) .
$$

This completes the proof.

\section{References}

[1] A. R. Ashrafi and G. H. Fath-Tabar, Bounds on the Estrada index of ISR $(4,6)$-fullerenes, Appl. Math. Lett. 24 (2011), 337-339, doi:10.1016/j.aml.2010.10.018, http://dx. doi. org/10.1016/j.aml.2010.10.018.

[2] S. K. Ayyaswamy, S. Balachandran, Y. B. Venkatakrishnan and I. Gutman, Signless Laplacian Estrada index, MATCH Commun. Math. Comput. Chem. 66 (2011), 785-794.

[3] A. T. Balaban, J. Đurđević and I. Gutman, Comments on $\pi$-electron conjugation in the five-membered ring of benzo-derivatives of corannulene, Polycyclic Aromatic Compounds 29 (2009), 185-208, doi:10.1080/10406630903087784.

[4] H. Bamdad, F. Ashraf and I. Gutman, Lower bounds for Estrada index and Laplacian Estrada index, Appl. Math. Lett. 23 (2010), 739-742, doi:10.1016/j.aml.2010.01.025, http: / / dx . doi.org/10.1016/j.aml.2010.01.025.

[5] R. Binthiya and P. B. Sarasija, On the signless Laplacian energy and signless Laplacian Estrada index of extremal graphs, Appl. Math. Sci. (Ruse) 8 (2014), 193-198, doi:10.12988/ams.2014. 311621.

[6] J. A. Bondy and U. S. R. Murty, Graph theory with applications, American Elsevier Publishing Co., Inc., New York, 1976.

[7] D. Cvetković, P. Rowlinson and S. Simić, An introduction to the theory of graph spectra, volume 75 of London Mathematical Society Student Texts, Cambridge University Press, Cambridge, 2010 . 
[8] D. Cvetković, P. Rowlinson and S. K. Simić, Signless Laplacians of finite graphs, Linear Algebra Appl. 423 (2007), 155-171, doi:10.1016/j.laa.2007.01.009, http: / / dx.doi.org/ $10.1016 / j .1 a a .2007 .01 .009$.

[9] J. R. Dias, Structure/formula informatics of isomeric sets of fluoranthenoid/fluorenoid and indacenoid hydrocarbons, Journal of mathematical chemistry 48 (2010), 313-329, doi:10.1007/ s10910-010-9671-9.

[10] Z. Du, B. Zhou and R. Xing, On maximum Estrada indices of graphs with given parameters, Linear Algebra Appl. 436 (2012), 3767-3772, doi:10.1016/j.laa.2011.12.031, http: / / dx . doi.org/10.1016/j.1aa.2011.12.031.

[11] E. Estrada, Characterization of 3d molecular structure, Chemical Physics Letters 319 (2000), 713-718, doi:10.1016/S0009-2614(00)00158-5.

[12] E. Estrada, Characterization of the folding degree of proteins, Bioinformatics 18 (2002), $697-$ 704, doi:10.1093/bioinformatics/18.5.697.

[13] G. H. Fath-Tabar, A. R. Ashrafi and I. Gutman, Note on Estrada and L-Estrada indices of graphs, Bull. Cl. Sci. Math. Nat. Sci. Math. (2009), 1-16.

[14] R. Grone and R. Merris, The Laplacian spectrum of a graph. II, SIAM J. Discrete Math. 7 (1994), 221-229, doi:10.1137/S0895480191222653, http://dx.doi.org/10.1137/ S0895480191222653.

[15] R. Grone, R. Merris and V. S. Sunder, The Laplacian spectrum of a graph, SIAM J. Matrix Anal. Appl. 11 (1990), 218-238, doi:10.1137/0611016, http: / /dx.doi .org/10.1137/ 0611016.

[16] I. Gutman, H. Deng and S. Radenković, The Estrada index: an updated survey, Zb. Rad. (Beogr.) 14(22) (2011), 155-174.

[17] I. Gutman and J. Đurđević, Fluoranthene and its congeners—a graph theoretical study, MATCH Commun. Math. Comput. Chem. 60 (2008), 659-670.

[18] I. Gutman and J. Đurđević, On $\pi$-electron conjugation in the five-membered ring of fluoranthene-type benzenoid hydrocarbons, J. Serb. Chem. Soc 74 (2009), 765-771, doi: 10.2298/JSC0907765G.

[19] I. Gutman and J. Đurđević, Cycles in dicyclopenta-derivatives of benzenoid hydrocarbons, MATCH Commun. Math. Comput. Chem. 65 (2011), 785-798.

[20] A. Khosravanirad, A lower bound for Laplacian Estrada index of a graph, MATCH Commun. Math. Comput. Chem. 70 (2013), 175-180.

[21] J. Li and J. Zhang, On the Laplacian Estrada index of unicyclic graphs, MATCH Commun. Math. Comput. Chem. 68 (2012), 835-842.

[22] R. Merris, Laplacian matrices of graphs: a survey, Linear Algebra Appl. 197/198 (1994), 143176, doi:10.1016/0024-3795(94)90486-3, second Conference of the International Linear Algebra Society (ILAS) (Lisbon, 1992), http://dx.doi.org/10.1016/0024-3795 (94) $90486-3$.

[23] W.-H. Wang, Unicyclic graph with maximal Estrada indices, MATCH Commun. Math. Comput. Chem. 68 (2012), 939-955.

[24] B. Zhou and I. Gutman, More on the Laplacian Estrada index, Appl. Anal. Discrete Math. 3 (2009), 371-378, doi:10.2298/AADM0902371Z, http://dx.doi.org/10.2298/ AADM0 902371 Z.

[25] B.-X. Zhu, On the Laplacian Estrada index of graphs, MATCH Commun. Math. Comput. Chem. 66 (2011), 769-776. 